\title{
Control of a Hypersegmented Space Telescope
}

\author{
Douglas G. MacMynowski* \\ California Institute of Technology, Pasadena, California 91125 \\ DOI: $10.2514 / 1.55428$
}

\begin{abstract}
The primary mirror diameter of affordable space telescopes is limited by mass and manufacturing cost. Currently planned optical/near-infrared space telescopes use a segmented primary mirror with relatively few segments and make limited use of real-time position control. However, control can be used as an enabler for a fundamentally different, very highly segmented architecture, leading to a significant reduction in areal density, and hence a significant increase in the realistically achievable diameter of a space telescope. Weight can be reduced by minimizing the structure that supports mirror segments and instead relying on control for overall stiffness. Furthermore, smaller segments can be thinner (hence, lighter) while still providing sufficient internal rigidity. However, with these architectural changes, the control problem involves not only thousands of actuators and sensors but also many lightly damped modes within the control bandwidth. The objective here is to demonstrate that this control problem is solvable by applying a local control approach. This is illustrated for a 30-m-diam primary mirror composed of 12,000 0.3-m-diam segments.
\end{abstract}

\section{Introduction}

$\mathbf{T}$ ELESCOPES in space allow science in wavelength bands absorbed by Earth's atmosphere avoid atmospheric turbulence, provide a stable environment with low infrared (IR) background noise, and permit long integration times [1]. However, cost is a significant driver. Ground-based optical telescopes are currently being designed with segmented primary mirror diameters of $30 \mathrm{~m}$ (using 492 segments) [2] and $39 \mathrm{~m}$ (798 segments) [3]. In contrast, the 18-segment primary mirror of the James Webb Space Telescope (JWST) [4] is $6.5 \mathrm{~m}$, and future designs for the Advanced Technology Lärge Aperture Space Telescope (ATLAST) range from a monolithic $8 \mathrm{~m}$ mirror to segmented 9.2 and $16.8 \mathrm{~m}$ designs [1] , all significantly smaller than what can be achieved at much lower cost on the ground. The goal herein is to propose active control of the mirror segments as a key enabler for building much larger diameter telescopes in space.

A key driver limiting affordable aperture sizes in space is the primary mirror mass or areal density, with the JWST and ATLAST segmented mirrors being of order $30 \mathrm{~kg} / \mathrm{m}^{2}$ (including the structure that supports the mirror segments) [5]. In contrast, actuated hybrid silicon carbide mirror segments have been proposed [6] with areal densities less than $10 \mathrm{~kg} / \mathrm{m}^{2}$, as low as $3 \mathrm{~kg} / \mathrm{m}^{2}$ using 1 -mm-thick silicon segments [7], and potentially even thinner and lighter with internal actuation [8]. However, highly segmented space telescope designs such as that presented by Dekany et al. [7] (using $\sim 0.3$-m-diam segments) have not included controls analysis, and the aim here is to illustrate that the resulting large numbers of segments (e.g., 12,000 for a $30 \mathrm{~m}$ mirror) can be robustly controlled. There are of course additional design and manufacturability issues regarding the segments, actuation, and sensing. Highly segmented concepts have also been introduced for ground-based telescopes [9], and other space telescope concepts with 1000 or more small segments have been proposed [10]. Membrane-based approaches have also been suggested for large lightweight space telescopes (see, e.g., the review by Santer and Seffen [11]); however, these may also require somewhat similar controls technology to provide adequate optical surface quality.

Received 25 June 2011; revision received 24 September 2011; accepted for publication 26 September 2011. Copyright $@ 2011$ by the American Institute of Aeronautics and Astronautics, Inc. All rights reserved. Copies of this paper may be made for personal or internal use, on condition that the copier pay the $\$ 10.00$ per-copy fee to the Copyright Clearance Center, Inc., 222 Rosewood Drive, Danvers, MA 01923; include the code 0731-5090/12 and \$10.00 in correspondence with the CCC.

*Senior Research Associate, Control and Dynamical Systems; macmardg@cds.caltech.edu.
While some mechanical interface is required between neighboring segments to provide in-plane stiffness, providing a stiff backsupport structure would defeat the purpose of minimizing the total mass. Minimizing structural mass results in a mirror that is highly flexible in its optically relevant out-of-plane motion, and active surface control will be required: even though there are few disturbances in space, some stiffness is required to enable repointing of the telescope in reasonable time. Providing this stiffness with active control requires a control bandwidth that includes some of the structural modes; the control approach must therefore account for both thousands of actuators and sensors and many lightly damped structural modes within the control bandwidth. A solution for this problem has recently been proposed in the context of large (1-3 m) deformable mirrors for ground-based adaptive optics $[12,13]$, and similar ideas are applied here to the control of a segmented space telescope.

The first important observation is that it is always possible to implement collocated rate feedback with some minimum but finite bandwidth [12]; this is essential for implementing control with realistic (finite bandwidth) actuators and sensors. The bandwidth needs to be high enough so that there is high modal overlap when the control rolls off; this frequency is lower when the modal density is high. Significant damping can be added, making position control more straightforward. Second, collocated control guarantees robustness but does not provide adequate stiffness for low-spatialfrequency deformations. Feedback of global sensor information can provide performance, but it has the potential for poor robustness because it relies on a global model (i.e., how every actuator affects every sensor). The strategy proposed herein uses only local model and sensor information: in response to some particular segment motion, a distribution of actuator commands is applied to both that segment and its neighboring segments rather than either a purely collocated actuator command (which leads to excitation of global modes) or a global distribution (which depends on a global model). A similar strategy was shown for deformable-mirror control to yield performance near that of a global solution while retaining robustness by not relying on global information [12]. No claim is made here regarding optimality but simply a demonstration of feasibility.

While the objective here is to demonstrate that a large array of segments can be controlled, and not to provide a detailed point design of a telescope using these ideas, a general description is useful for defining design constraints (Sec. II). The remaining sections develop the telescope model (Sec. III), and develop and demonstrate the local control (Sec. IV).

\section{Concept}

Science cases for future large-aperture optical and near-IR space telescopes are described, for example, in Postman et al. [1]. One 
motivation for such a facility would be the capability for assessing the potential for life on Earth-like planets of other stars. Larger apertures allow a greater number of star systems to be considered, both because more light-gathering capability means less integration time and because higher resolution allows distinguishing star light from planet at greater distances from the Earth. For this type of science, the overall field of view would not need to be very large. Sufficient integration time is required for spectroscopic measurements to understand atmospheric composition, while the total number of targets to be evaluated by the facility during its lifetime might be only a few thousand. For this mission, then, it may be acceptable if it takes many hours to change the orientation of the telescope to point at a different target, but a settling time of days would impact the science mission. For the simulation parameters described in the next section, the first resonant frequency of a $30 \mathrm{~m}$ primary mirror is $\sim 0.1 \mathrm{~Hz}$. The damping of space structures can be as low as $0.1 \%$, giving an uncontrolled settling time of order a day from a 90 deg slew maneuver to a few nanometer residual error.

The key innovation herein is thus a strategy that enables control of a highly segmented filled-aperture primary mirror. An example is illustrated in Fig. 1, with $N=12,000$ hexagonal segments with a maximum radius of $0.15 \mathrm{~m}$ (as in Dekany et al. [7]). The detailed optical design would depend on the science mission and engineering optimization, and it is not important for developing and demonstrating an approach for controlling a hypersegmented primary mirror. Mass is minimized both by 1) reducing segment size so that segment thickness can be reduced, without requiring additional degrees of freedom of actuation internal to the segment for shape control; and 2) minimizing the mechanical interconnections between segments. In-space assembly, either robotic or with astronauts, is plausible if the interconnection tasks are straightforward $[10,14,15]$ and if control can be used to correct errors resulting from not having a precision deployable structure [6].

The mechanical interface between neighboring segments provides stiffness for the in-plane degrees of freedom of the segment array, but it does not need to provide significant stiffness for the out-of-plane degrees of freedom, since these will need to be actively controlled. A single interface between segments, as shown in Fig. 2, simplifies assembly, but it requires that the interface provide stiffness and actuation authority for both the relative out-of-plane intersegment motion and the intersegment dihedral angle (relative rotation along the segment edge). Note that, with only a single degree of freedom of actuation per intersegment edge, there would not be enough actuators to constrain the $3 \mathrm{~N}$ degrees of freedom of the full $\mathrm{N}$-segment array; with two degrees of freedom of actuation per edge, the system is controllable, with more actuators than required to control segment rigid-body motion. It is also possible to use two interconnects [7]; the

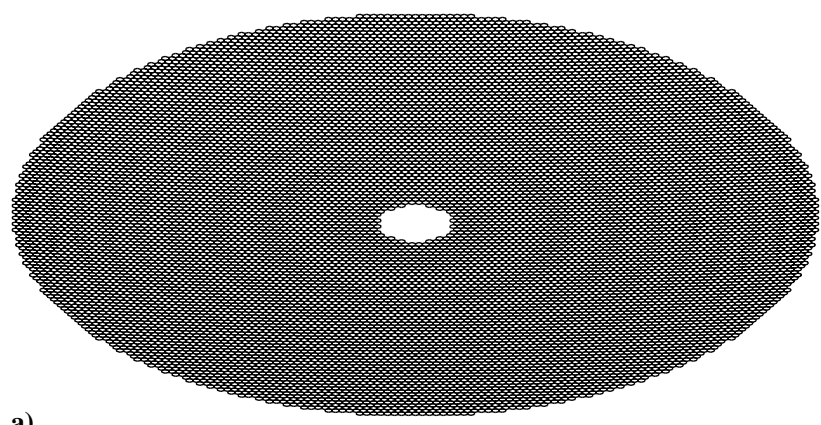

a)

E. $600 \mathrm{~m}$

$\mathrm{M} 2$

b)

Fig. 1 Schematic of telescope design: a) 12,000 segment mirror (viewed from a $30 \mathrm{deg}$ angle), with segment diameter $1 \%$ of the mirror diameter (e.g., $30 \mathrm{~m}$ with $0.3 \mathrm{~m}$ segments). The secondary mirror (M2) would be on a separate formation-flying spacecraft, of order $600 \mathrm{~m}$ away, as shown in Fig. 1b, with the primary mirror (M1) to scale.

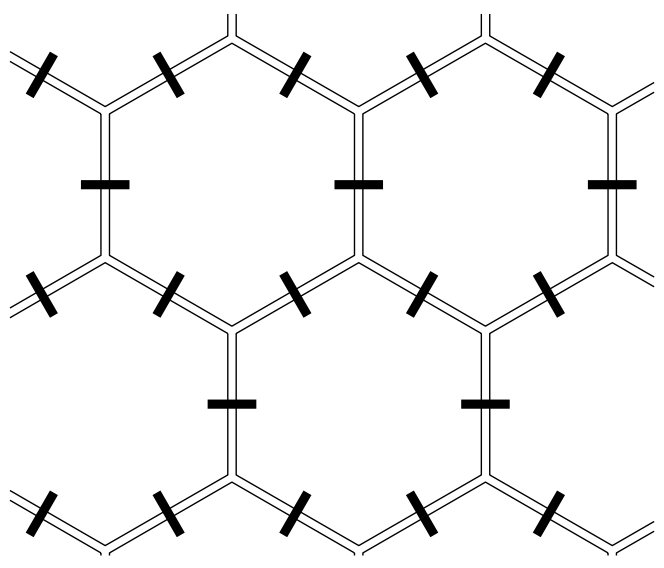

Fig. 2 Detail on segmentation geometry (with gaps enlarged for clarity), using a single mechanical interconnection between neighboring segments.

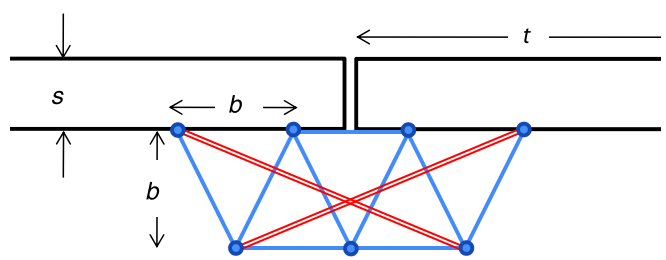

Fig. 3 Sketch of mechanical interconnect between neighboring segments used to estimate representative stiffness values in simulation. The long diagonals (double lines) are active members to apply differential force/torques between segments.

relative advantages are unclear without more detail design, including consideration for both actuation and assembly. The single interconnect approach is used herein, with nominal stiffness parameters based on the sketch in Fig. $\underline{3}$.

In addition to eliminating the structural weight supporting the primary mirror (M1), there is no need for structure between the primary and secondary mirrors (M2) if formation flying is used for the M2 subsystem, as suggested by Burge et al. [16] or Dekany et al. [7] (and plausible if repointing of the telescope is not frequent). This also means that the primary focal length of the telescope is not driven by structural weight or launch-packaging considerations but only by the optical design; Burge et al. [16] proposed a focal ratio of 20 so that, for a $30 \mathrm{~m}$ primary mirror, M2 would be $0.6 \mathrm{~km}$ away. A consequence of long focal lengths and many segments is that the segment surface can be spherical rather than hyperbolic; hence, the segments can be identical: this is essential for minimizing manufacturing cost and also reduces the complexity of in-space assembly. The overall M1 shape may still be parabolic or hyperbolic, and optical distortion may be improved with the ability to statically adjust the radius of curvature of each segment; the required adjustment decreases as the segment size decreases. Sun shades can also be separate satellites flown in formation [16]. Formation flying requires that the telescope not be in Earth orbit with its gravitygradient torques but at one of the stable Lagrange points (as JWST). The mass of the spacecraft bus and instrumentation, located in the central obscuration of the primary mirror, will be significant, but does not scale with the collecting area.

\section{Model}

Since the in-plane segment motions are passively constrained by the mechanical interconnection, only the three out-of-plane degrees of freedom need to be included in the dynamic model; here, we describe the motion using segment piston $(z)$ and rotations (Fig. 4) so the segment motion is described by $x_{i}=\left[\begin{array}{lll}\phi_{i} & \theta_{i} & z_{i}\end{array}\right]^{T}$, and the generalized displacement vector for the full mirror $x=\left[x_{1}^{T} \ldots x_{N}^{T}\right]^{T}$ satisfies 


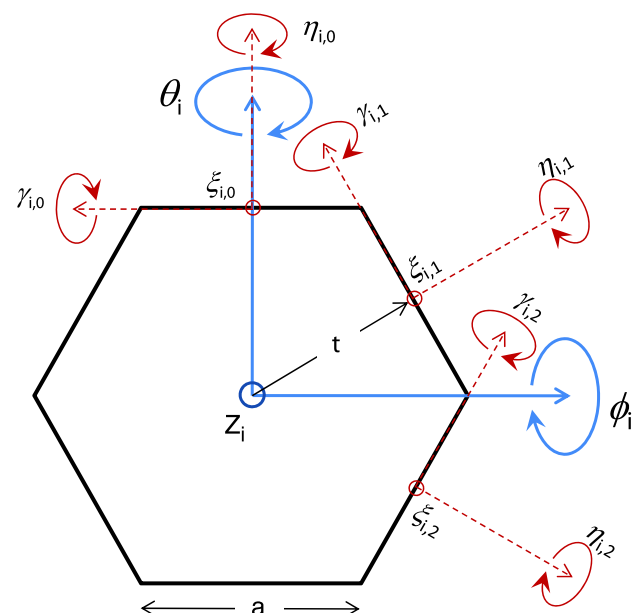

Fig. 4 Segment coordinate system $\theta_{i}, \phi_{i}$, and $z_{i}$ (thick lines) and rotated coordinate systems $\eta_{i, k}, \gamma_{i, k}$, and $\xi_{i, k}$ at the interface point with the neighboring segment in the $k$ th direction, shown for $k=0,1$, and 2 only.

$$
M \ddot{x}+D \dot{x}+K x=\Phi u
$$

for global mass, damping, and stiffness matrices $M, D$, and $K$; the vector of actuator forces and moments $u$; and the corresponding influence matrix $\Phi$.

For ease of calculation, an additional coordinate system (Fig. 4) is introduced for each connection point $k=0, \ldots 5$ on segment $i$ :

$$
\left(\begin{array}{l}
\eta_{i, k} \\
\gamma_{i, k} \\
\xi_{i, k}
\end{array}\right)=T_{k} x_{i}
$$

where the transformation matrix is

$$
T_{k}=\left(\begin{array}{ccc}
\sin (k \pi / 3) & \cos (k \pi / 3) & 0 \\
-\cos (k \pi / 3) & \sin (k \pi / 3) & 0 \\
t \cos (k \pi / 3) & -t \sin (k \pi / 3) & 1
\end{array}\right)
$$

where $t=a \sqrt{3} / 2$ is the radius at the interconnection points, and $a$ is the segment radius or side length. The notation $j=i(k)$ will be used to refer to the segment $j$ that borders segment $i$ at orientation $k$.

The mass matrix for an individual segment with mass $m_{s}$ and moments of inertia $J_{\phi}$ and $J_{\theta}$ is

$$
M_{s}=\left(\begin{array}{ccc}
J_{\phi} & 0 & 0 \\
0 & J_{\theta} & 0 \\
0 & 0 & m_{s}
\end{array}\right)
$$

The connection joint stiffness introduces forces proportional to the differential motion across the joint, i.e., for relative rotation $\eta$ (about the line between the segments); relative rotation $\gamma$ (the dihedral angle between the segments); and relative edge height $\xi$, with stiffnesses $K_{\eta}, K_{\gamma}$, and $K_{\xi}$, respectively. For the interconnection used here, $K_{\eta}$ will be small and could be ignored. Note that, in Eq. (6), the rotation $\eta_{i, k}$ on segment $i$ and $\eta_{i(k), \bmod (k+3,6)}$ on the neighboring segment across a joint have an opposite sign convention due to the rotated reference frame. The differential rotation is thus the sum $\eta_{i, k}+$ $\eta_{i(k) \bmod (k+3,6)}$, and similarly for $\gamma$. The stiffness matrix for each connection joint is

$$
K_{s}=\left(\begin{array}{ccc}
K_{\eta} & 0 & 0 \\
0 & K_{\gamma} & 0 \\
0 & 0 & K_{\xi}
\end{array}\right)
$$

Actuation could be introduced to act on all three motions at each joint, but sufficient actuation degrees of freedom require only two. A differential force $F$ and a differential torque $M$ acting in the $\gamma$ direction are used here. Actuators at three of the six interconnection points $(k=0,2,4)$ are numbered with that segment, and the other three $(k=1,3,5)$ are numbered with the relevant neighbor. The sign convention is chosen so that each actuator produces a positive influence on the local coordinate of the segment it is numbered with and an equal and opposite force or moment on the neighboring segment. Again, the sign change for $\gamma$ that results from the rotated reference frame on the neighboring segment needs to be taken into account.

Neglecting damping, the equations of motion for each segment can now be written by including the stiffness and actuation contributions in the local coordinate frame as

$$
\begin{array}{r}
M_{s} \ddot{x}_{i}+\sum_{k=0}^{5} T_{k}^{T} K_{s}\left[\left(\begin{array}{c}
\eta_{i, k} \\
\gamma_{i, k} \\
\xi_{i, k}
\end{array}\right)-\left(\begin{array}{c}
-\eta_{i(k), \bmod (k+3,6)} \\
-\gamma_{i(k), \bmod (k+3,6)} \\
\xi_{i(k), \bmod (k+3,6)}
\end{array}\right)\right] \\
=\sum_{k=0,2,4} T_{k}^{T}\left(\begin{array}{c}
0 \\
M_{i, k} \\
F_{i, k}
\end{array}\right)-\sum_{k=1,3,5} T_{k}^{T}\left(\begin{array}{c}
0 \\
-M_{i(k), k} \\
F_{i(k), k}
\end{array}\right)
\end{array}
$$

where the relevant stiffness and actuation contributions are not included at boundary segments. The equations of motion are sparse; to preserve this, damping is added of the form $D=c_{1} M+c_{2} K$, with $c_{1}$ and $c_{2}$ chosen to give $0.1 \%$ damping at the first resonance and at $500 \mathrm{~Hz}$.

The spacecraft bus and instrumentation in the center of the mirror will have significant mass $M_{\text {center }}$. The model assumes that the bus is connected to the neighboring segments with the same mechanical interconnection as between any other pair of segments. The internal flexibility of the bus is ignored.

For the purposes of illustrating the control concept, consider silicon carbide ( $\mathrm{SiC})$ segments, with $s=1 \mathrm{~mm}$ thick and with maximum radius $a=15 \mathrm{~cm}$. For analysis, the mechanical interconnections, actuators, sensors, and electronics/cabling are assumed to increase the mass $\rho s=2.95 \mathrm{~kg} / \mathrm{m}^{2}$ of the $\mathrm{SiC}$ alone by $50 \%$ without altering the mass distribution, captured by the mass fraction $\mu=1.5$. Thus, the segment mass and moment of inertia are

$$
m=\mu \frac{3 \sqrt{3}}{2} \rho s a^{2}, \quad J_{\theta}=J_{\phi}=\mu \frac{5 \sqrt{3}}{16} \rho s a^{4}
$$

Representative values for the stiffness of the mechanical interconnection between segments are obtained by analyzing the truss structure in Fig. 3 ; this is only to obtain reasonable parameter estimates and not to propose a specific design. The mass of each mechanical interconnect is assumed to be $10 \%$ of the mass of the $\mathrm{SiC}$ segment (so the total mechanical mass adds $30 \%$ to the mass). The depth $b$ influences the stiffness; this is chosen to be a fraction of the segment radius to allow a design that folds into the back of the segment for launch. This yields the stiffness parameters in Table 1. Using these parameters, the first resonance of an individual segment will be of order $1 \mathrm{kHz}$; for the purpose of understanding the low-spatial/temporal frequency modes of the overall mirror, the flexibility of an individual segment can be ignored.

The modal density of the entire mirror array, without these internal flexible modes of each segment, is roughly constant, as shown in Fig. 5. The first flexible mode of the full segment array is at $0.13 \mathrm{~Hz}$, and there are 14 flexible modes below $1 \mathrm{~Hz}$.

Table 1 Parameters used in simulation

\begin{tabular}{cccc}
\hline \hline Parameter & Value & Parameter & Value \\
\hline$\rho$ & $2.95 \times 10^{3} \mathrm{~kg} / \mathrm{m}^{3}$ & $K_{\eta}$ & 10 \\
$a$ & $0.15 \mathrm{~m}$ & $K_{\gamma}$ & $4.2 \times 10^{3} \mathrm{Nm} / \mathrm{rad}$ \\
$s$ & $1 \mathrm{~mm}$ & $K_{\xi}$ & $3.7 \times 10^{6} \mathrm{~N} / \mathrm{m}$ \\
$b$ & $0.04 \mathrm{~m}$ & $M_{\text {center }}$ & $4000 \mathrm{~kg}$ \\
$N$ & 12,000 & $J_{\text {center }}$ & $3100 \mathrm{~kg} \mathrm{~m}{ }^{2}$ \\
\hline \hline
\end{tabular}




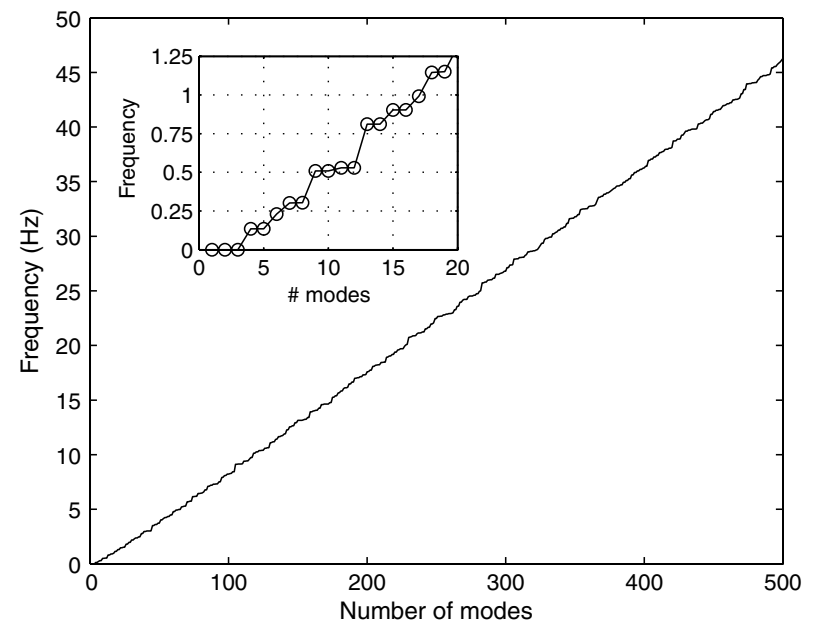

Fig. 5 First 500 resonant frequencies of a $30 \mathrm{~m}$ mirror using the segment parameters in Table 1 . The inset shows the first 20 modes.

\section{Control}

\section{A. Control Problem}

There are $3 N$ controlled degrees of freedom for the overall segmented mirror (the in-plane degrees of freedom are passively controlled by the mechanical interconnection) and slightly less than $6 \mathrm{~N}$ degrees of freedom of relative actuation (there is no actuation on segment edges at the outer boundary of the mirror). The extra degrees of freedom of actuation could either be constrained to be zero (as here) or used to provide small deformations of the segments to modify their radius of curvature in both the radial and azimuthal directions.

Two sensors are also needed on each edge to measure the relative motion between segments, as on ground-based segmented-mirror telescopes [17], where differential height between segments can be measured with a resolution of a few nanometers using either differential capacitive [18] or differential inductive sensors [19]. Unless a manufacturing approach is used that ensures sensor installation errors of nanometers, an initial phasing approach using star light would be needed after the mirror was assembled in order to determine the correct set point for each sensor; these techniques are well established on the ground, but some modifications to the approach would be required to handle many thousands of segments [6]. Having mechanical edge sensors means that optical feedback is not continuously required, except perhaps for low-order mirror deformations that are not as well observed by relative measurements between segments.

The transformation between segment motion and sensor response can be determined from geometry [20]. The global piston, tip, and tilt of the entire mirror cannot be measured with internal relative sensor measurements (nor controlled with relative actuators); aside from these degrees of freedom, the transformation is invertible. However, while the transformation from motion to sensor response is sparse, the inverse is in general fully populated; that is, the estimation of segment motion requires global knowledge. For ground-based segmented mirrors, where the actuators influence the absolute motion of the segments, the inverse transformation has been shown to require attention to robustness (which is challenging [21]) and to computation (which is solvable [22,23]). With actuation that influences only relative motion between segments, there is a related problem of finding the set of actuator commands that produces a desired segment motion. Here, the focus is on dynamics (in Sec. IV.B) and on this actuator distribution (or spatial response) problem (in Sec. IV.C), and it is assumed that the estimation problem is solvable using the previously developed techniques cited above.

Figures $\underline{6}$ and 7 illustrate representative characteristics of the mirror, including the transfer function between a representative actuator and the collocated response, and the static response shape of the mirror resulting from a unit command on a representative actuator. The dynamics and spatial response issues noted above are
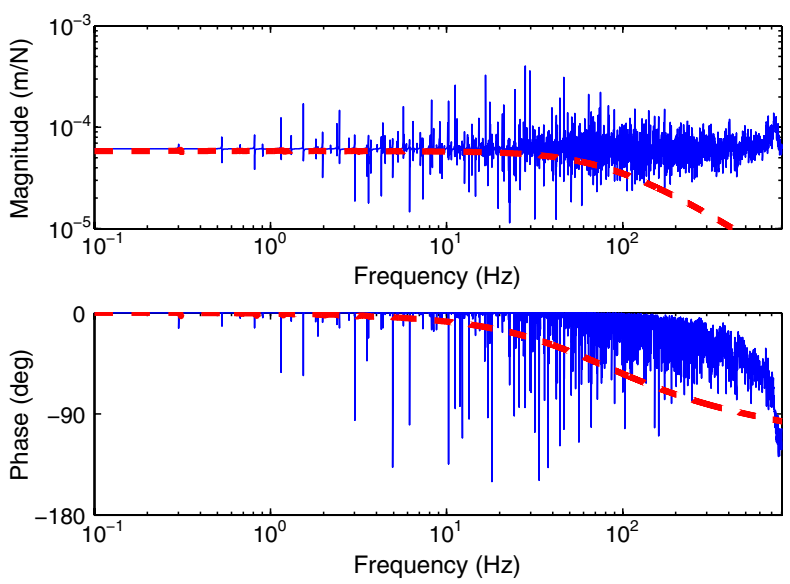

Fig. 6 Representative collocated transfer function for a force actuator, without and with (dashed) active damping.

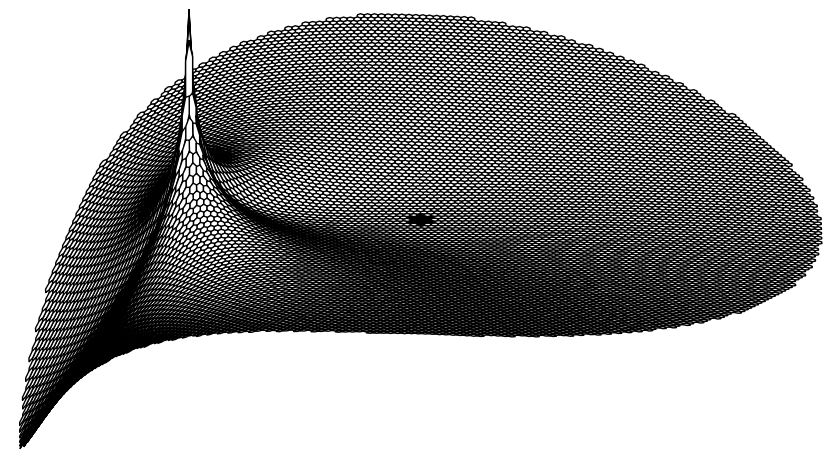

Fig. 7 Static response pattern for a torque command on a representative actuator (magnified); the influence pattern is nonlocal.

related to the behavior in these two figures. The first challenge is the ability to add active damping despite the presence of many modes both within and above the control bandwidth (i.e., compensating for the behavior in Fig. 6). Realistic actuator/sensor dynamics preclude arbitrarily high bandwidth and rolloff will be required at some frequency. The second challenge is the ability to manage the wide range of spatial scales introduced by actuation without requiring global knowledge that would inevitably limit robustness (i.e., compensating for the resonant behavior in Fig. 7). Approaches for these key challenges are described in Secs. IV.B and IV.C.

\section{B. Collocated Control}

The relative motion collocated with the actuators can be obtained from sensors between neighboring segments, using only a local transformation. Using this information, collocated rate feedback can add significant damping, making the position control design more straightforward. While this is guaranteed to be robustly stable, in practice, rate feedback has finite bandwidth due to sensor and actuator dynamics and electronic implementation. While the simulation here has a highest resonant frequency $\sim 1.5 \mathrm{kHz}$, the real mirror will have higher frequency resonances due to internal segment dynamics. However, above some frequency $f_{\text {ac }}$, the half-power bandwidth of the structural modes $\left(2 \zeta f_{m}\right.$ for open-loop damping $\zeta$ and modal frequency $f_{m}$ ) will exceed the modal spacing (roughly $0.1 \mathrm{~Hz}$ for the simulation parameters here; see Fig. 5). This leads to a relatively smoother transfer function in both magnitude and phase [12]. See Fig. 6; where the variation in phase and magnitude becomes smaller above roughly $500 \mathrm{~Hz}$. Practically, this means that active damping is feasible, provided it has a minimum bandwidth of at least this acoustic limit of $f_{\text {ac }}$. The high number of modes in this problem means that the acoustic limit for the structural behavior is at a low enough frequency that active damping is plausible with realistic actuator and sensor bandwidths (less than $1 \mathrm{kHz}$ ). 
Collocated position feedback behaves as an electronic spring, and it is similarly guaranteed to be stable but with negligible phase margin if the gain is large enough to have a significant effect (loop gain larger than 1). A small, stable, position gain is used in the simulations here, which has a small stiffening effect on the lowest frequency modes.

The average slope of the transfer function from force to collocated displacement, or torque to collocated rotation, is roughly zero (a constant; Fig. 6); thus, rate feedback has a higher loop gain at higher frequency, and thus adds more damping to higher frequency modes than to low. A high rate feedback gain is chosen here that results in overdamping many of the high-frequency modes in order to provide some damping to the lower frequency modes. Note that even adding a small amount of damping at each collocated actuator/sensor pair can result in significant total damping due to the large number of actuators. Including both rate and position feedback, the collocated loop transfer function for a representative actuator is shown in Fig. 8 , and the resulting closed-loop transfer function is shown in Fig. $\underline{\underline{\sigma}}$. [The curve with active damping diverges from the uncontrolled case at high frequency because the damping parameters are chosen so that the high-frequency modes are overdamped (yielding real rather than complex poles).] The added damping yields a more straightforward problem for an outer control loop.

\section{Local Feedback}

The control has higher gain on low-spatial-frequency deflection patterns than on high (that is, the plant is ill-conditioned); this is evident in the static response pattern in Fig. 7, where a high-spatialfrequency input leads to excitation of both high- and low-spatial frequencies. A collocated strategy means that, in response to a particular nonzero intersegment motion, the control will apply solely the corresponding relative actuator command, giving a global response to a local error. A global feedback strategy could readily avoid this by inverting the system dynamics, at the expense of requiring, and hence being dependent on, both global model knowledge and global state information. The innovation used herein is to use only local actuation in response to a particular segment displacement; this provides a remarkably good compromise between performance and required knowledge [12,13]. Because the collocated control described above suppresses much of the dynamic variation, it is sufficient to develop an approximate local inverse that is valid quasi statically.

The resulting architecture is shown in Fig. 9. $G(s)$ is the plant dynamics from actuator input to collocated sensor output (including any required local transformation from sensor to equivalent collocated response). Collocated rate feedback $K_{v}$ adds damping and position gain $K_{p}$ adds stiffness, but both are limited by actuator and
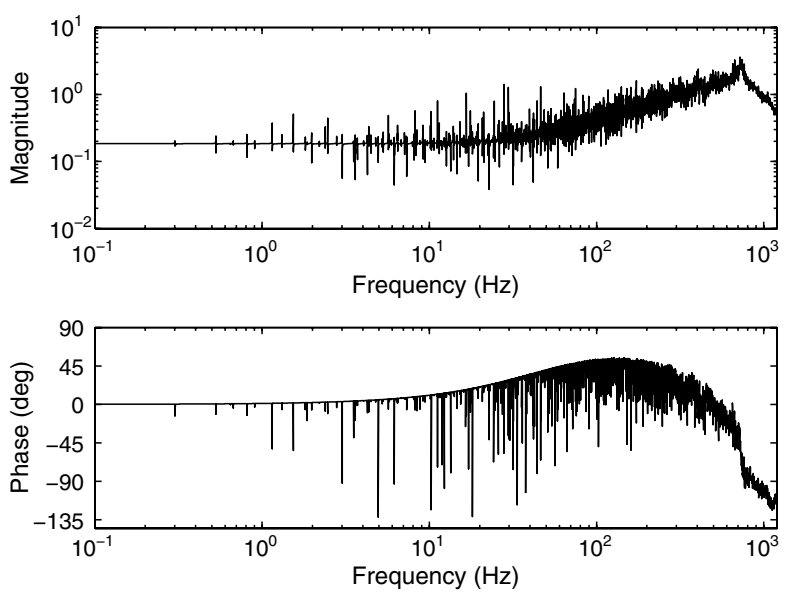

Fig. 8 Representative collocated loop transfer function for a force actuator. Rolloff is introduced at high frequencies, but because the phase variations are small, the control remains stable. In addition to the active damping, a small position feedback adds some stiffness.

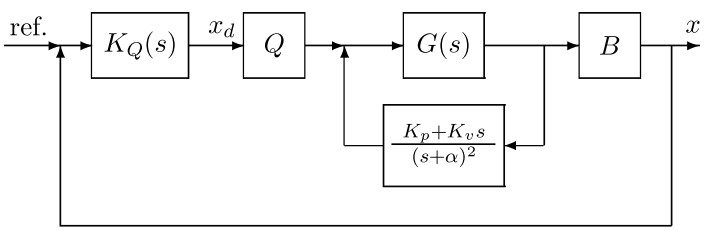

Fig. 9 Block diagram of control system.

sensor dynamics represented by poles at $s=-\alpha$. The matrix $B$ describes the problem of estimating segment positions from relative sensor information; this has received attention in the context of ground-based telescopes, and it is not discussed here. The remainder of this section describes the construction of the matrix $Q$ that provides a sparse, local approximate static inverse to $B G$, so that $B G(0) Q \simeq I ; Q$ takes desired segment position commands $x_{d}$ and generates an actuator distribution that approximately matches $x_{d}$ without requiring forces and moments throughout the entire mirror.

Define the set $\Omega_{k}$ of actuators local to segment $k$. For illustration here, $\Omega_{k}$ is chosen to include all actuators on both segment $k$ and every immediately adjacent segment, for a total of 60 (out of 72,000) actuators for an interior segment, as shown in Fig. 10. The number of actuators used in each set is a design choice that trades nominal performance with increased complexity and decreased robustness; this ultimately needs to be made based on specific design requirements. Increasing the size of the local region will result in improved suppression of more modes in the local control loop [13].

From Eq. (1), the static response of the mirror to an actuator command $u$ is $x=C \Phi u$, where $C=K^{\#}$ is a modified compliance matrix, and where the uncontrollable rigid-body modes are projected out ( $C$ is the pseudoinverse of $K$; note that eigenvectors corresponding to zero eigenvalues of $K$ are also uncontrollable). $C$ is fully populated and ill-conditioned.

Define vector $e_{k i}$ to be a unit displacement of coordinate $i$ on segment $k$, and choose the actuator response pattern $u_{k i}$ that minimizes the cost function

$$
J=\left\|C \Phi u_{k i}-e_{k i}\right\|_{2}
$$

subject to the constraint that elements of vector $u_{k i}$ not in the set $\Omega_{k}$ must be zero. That is, choose a local set of forces to minimize the rms error over the entire mirror in matching the displacement pattern. The constrained least-squares problem is equivalent to solving an unconstrained problem with a truncated matrix $\Psi_{k}=C \Phi_{: \Omega_{k}}$, where only the columns of $\Phi$ associated with actuators in $\Omega_{k}$ are retained. The row of the pseudoinverse of $\Psi_{k}$ corresponding to the $i$ th coordinate on the $k$ th segment gives the appropriate local actuator (force and moment) distribution $u_{k i}$ to compensate for an error at location $k$.

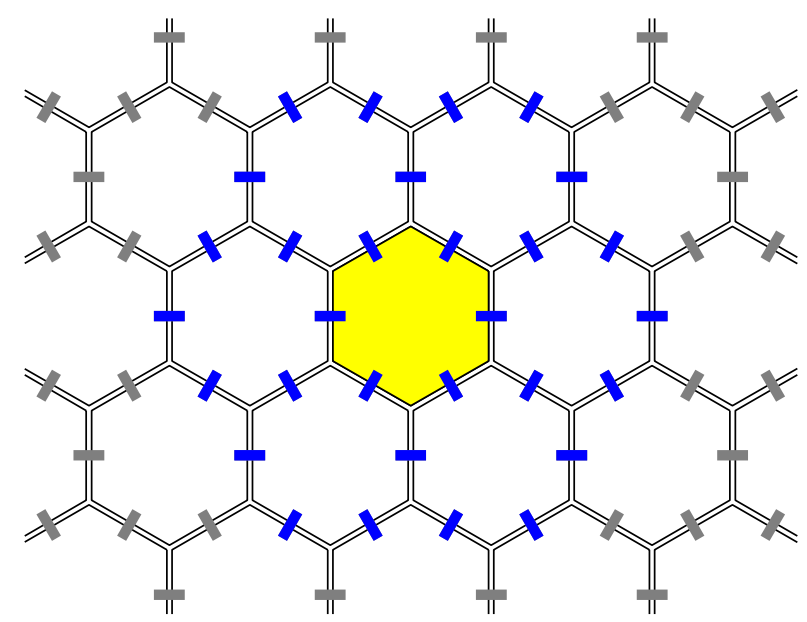

Fig. 10 Local set of actuators used in order to produce a desired motion of a single segment. This set involves 30 segment edges and 60 actuators; smaller or larger sets could be considered to trade off robustness and performance. 


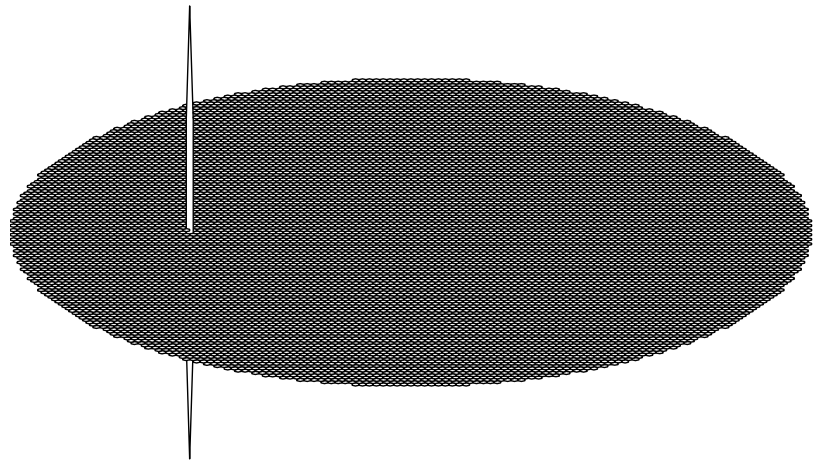

Fig. 11 Static response pattern for a command to a representative local family group in response to a desired single segment rotation; compare with Fig. . The response away from the segment is negligible.

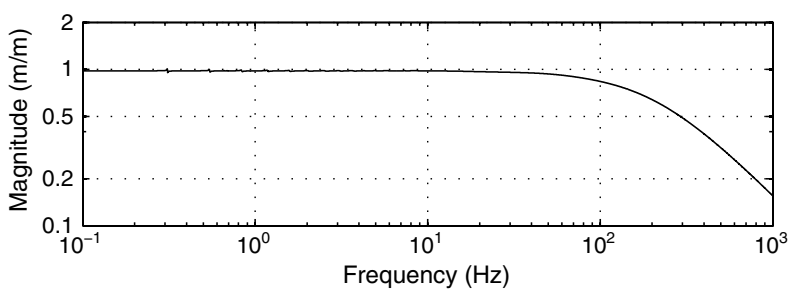

Fig. 12 Representative transfer function from an input to a local force distribution to the resulting segment position response (from one element of $x_{d}$ to the corresponding element of $x$ in Fig. 9).

By assembling, for each $k$ and $i$, the resulting pattern into a matrix $Q$ (so $Q_{\Omega_{k}, k i}=u_{k i}$ ), $Q$ gives an approximate inverse to the system at zero frequency, based on local actuation only. Control can then be based on $Q x$ rather than collocated control. This approach is identical in derivation to that in MacMynowski et al. [12], and it is motivated by local approaches for computationally efficient sparse reconstruction in adaptive optics estimation [24]. The resulting response distribution to a single segment rotation error is shown in Fig. 11; the response to a position error is similarly local.

A representative transfer function between an input to a single local actuator group and the resulting segment response is shown in Fig. 12; since $Q$ is an approximate static inverse of the plant, the resulting system is decoupled and normalized to unit gain at zero frequency. The crosscoupling to other displacements on the same segment are a factor of $10^{4}$ smaller. With this (static) transformation between segment position errors and the appropriate corresponding force distribution, it is much more straightforward to design a position controller.

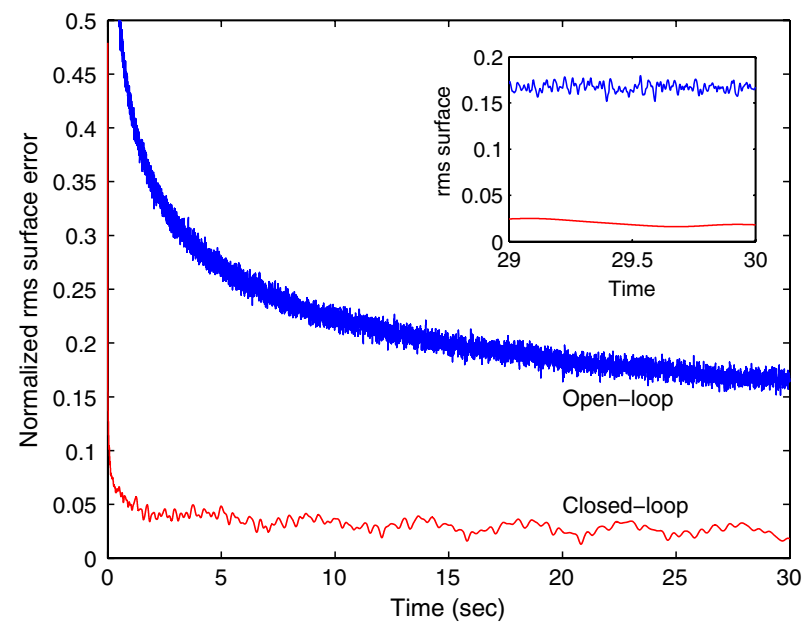

Fig. 13 Time history of rms surface response from a random initial condition, open-loop and closed-loop, both normalized relative to the rms surface error of the initial condition.
The resulting (normalized) closed-loop performance in response to an initial condition is shown in Fig. 13 using a low-bandwidth position controller. The initial condition included both high- and low-spatial frequencies. Further simulation and control optimization could involve more realistic disturbances, transient reorientation maneuvers, addition of sensor noise, etc. The highest spatial frequencies in the open-loop model are quickly damped due to the assumed nonmodal damping, leaving a broad range of spatial and temporal frequencies in the response (shown more clearly in the inset). The closed-loop case has a significant improvement immediately (the two cases start from the same initial condition) because mid- and high-frequency modes are overdamped. The residual response is from very low frequency modes that are not well damped by the control.

\section{Conclusions}

Active control is a potential enabler for future space telescopes with primary mirror areal densities an order of magnitude smaller than current generation telescopes, permitting an order of magnitude greater collecting area for the same launch weight. The reduced mass would be obtained by using very large numbers of identical small segments. Before such an architecture could be seriously considered for future missions, there are several challenges that must be overcome. A key challenge is the ability to control the resulting segment array, requiring control of thousands of degrees of freedom, with many lightly damped structural modes within the control bandwidth. Building off of recent research in controlling large flexible deformable mirrors, a control architecture for this problem is presented here; the approach combines collocated active damping with local position control.

First, real actuators and sensors have finite bandwidth; thus, any implementation of rate feedback to add damping will not be positive real above some frequency. However, there will always be some frequency at which the structure enters an acoustic limit, where the half-power bandwidth of any mode exceeds the modal spacing; thus, the phase excursions in the collocated transfer function decrease due to multiple modes being simultaneously excited. It is precisely because there are many structural modes in this problem that this frequency is not unrealistically high; hence, practical active damping is stable provided it has a minimum bandwidth.

Second, in addition to the wide range of temporal frequencies, there is a wide range of spatial frequencies excited by any actuator. With collocated feedback, a global response pattern is generated in response to a local position error. A global feedback strategy could correct this behavior, but requiring information from the entire mirror has the potential to introduce robustness problems. Instead, a local control strategy is introduced that does not depend on model or state knowledge far away from a given actuator. This is derived, not by considering what nearby information is necessary at each actuator location, but rather by what distribution of response is appropriate for any given local position error.

The strategy is demonstrated on a dynamic model of a $30 \mathrm{~m}$ mirror composed of 12,000 identical 30 -cm-diam segments. The main conclusion is that the ability to control the system is not a barrier to designing a space telescope with a large number of segments.

\section{Acknowledgment}

The simulation code used here was developed by Mattias Björklund, of the Royal Institute of Technology (KTH), Sweden.

\section{References}

[1] Postman, M., Brown, T., Sembach, K., Giavalisco, M., Traub, W., Stapelfeldt, K., Calzetti, D., Oegerle, W., Rich, R. M., Stahl, H. P., Tumlinson, J., Mountain, M., Soummer, R., and Hyde, T., "Science Drivers and Requirements for an Advanced Technology Large Aperture Space Telescope (ATLAST): Implications for Technology Development and Synergies with Other Future Facilities," Proceedings of the SPIE, Vol. 7731, Space Telescopes and Instrumentation, SPIE, Bellingham, WA, 2010, Paper 77312K. 
doi: $10.1117 / 12.857044$

[2] Nelson, J., and Sanders, G. H., "The Status of the Thirty Meter Telescope Project," Proceedings of the SPIE, Vol. 7012, Ground-Based and Airborne Telescopes 2, SPIE, Bellingham, WA, 2008, Paper 70121A.

doi: $10.1117 / 12.788238$

[3] Gilmozzi, R., and Spyromilio, J., "The 42 m European ELT: Status," Proceedings of the SPIE, Vol. 7012, Ground-Based and Airborne Telescopes 2, SPIE, Bellingham, WA, 2008, Paper 701219. doi:10.1117/12.790801

[4] Gardner, J. P., Mather, J. C., Clampin, M., Doyon, R., Greenhouse, M. A., Hammel, H. B., Hutchings, J. B., Jakobsen, P., Lilly, S. J., Long, K. S., Lunine, J. I., Mccaughrean, M. J., Mountain, M., Nella, J., Rieke, G. H., Rieke, M. J., Rix, H.-W., Smith, E. P., Sonneborn, G., Stiavelli, M., Stockman, H. S., Windhorst, R. A., and Wright, G. S., "The James Webb Space Telescope," Space Science Reviews, Vol. 123, 2006, pp. 485-606. doi:10.1007/s11214-006-8315-7

[5] Stahl, H. P., "Ares V Launch Vehicle Capability Enables Future Space Telescopes," Proceedings of the SPIE, Vol. 6687, SPIE, Bellingham, WA, 2007, Paper 66870L. doi: $10.1117 / 12.730853$

[6] Hickey, G., Barbee, T., Ealey, M., and Redding, D., "Actuated Hybrid Mirrors for Space Telescopes," Proceedings of the SPIE, Vol. 7731, Space Telescopes and Instrumentation, SPIE, Bellingham, WA, 2010, Paper 773120 doi: $10.1117 / 12.858217$

[7] Dekany, R. G., MacMartin, D. G., Chanan, G. A., and Troy, M., "Advanced Segmented Silicon Space Telescope (ASSiST)," Proceedings of the SPIE, Vol. 4849, Highly Innovative Space Telescope Concepts, SPIE, Bellingham, WA, 2002, pp. 103-111. doi: $10.1117 / 12.460563$

[8] Patterson, K., Pellegrino, S., and Breckinridge, J., "Shape Correction of Thin Mirrors in a Reconfigurable Modular Space Telescope," Proceedings of the SPIE, Vol. 7731, Space Telescopes and Instrumentation, SPIE, Bellingham, WA, 2010, Paper 773121. doi: $10.1117 / 12.861442$

[9] Padin, S., "Design Considerations for a Highly Segmented Mirror," Applied Optics, Vol. 42, No. 16, 2003, pp. 3305-3312. doi:10.1364/AO.42.003305

[10] Oegerle, W. R., Purves, L. R., Budinoff, J. G., Moe, R. V., Carnahan, T. M., Evans, D. C., and Kim, C. K., "Concept for a Large Scalable Space Telescope: In-Space Assembly," Proceedings of the SPIE, Vol. 6265, Space Telescopes and Instrumentation 1: Optical, Infrared, and Millimeter, SPIE, Bellingham, WA, 2006, Paper 62652C. doi:10.1117/12.672244

[11] Santer, M. J., and Seffen, K. A., "Optical Space Telescope Structures: The State of the Art and Future Directions," The Aeronautical Journal, Vol. 113, No. 1148, 2009, pp. 633-645.

[12] MacMynowski, D. G., Heimsten, R., and Andersen, T., "Distributed Force Control of Deformable Mirrors," European Journal of Control, Vol. 17, No. 3, 2011, pp. 249-260. doi: $10.3166 /$ ejc. $17.249-260$

[13] Heimsten, R., Owner-Petersen, M., Ruppel, T., MacMynowski, D. G., and Andersen, T., "Suppressing Low-Order Eigenmodes with Local Control for Deformable Mirrors," Optical Engineering, Vol. 51, No. 2, 2012.

[14] Doggett, W., "Robotic Assembly of Truss Structures for Space Systems and Future Research Plans," IEEE Aerospace Conference Proceedings, IEEE, New York, 2002, pp. 3589-3598.

[15] Watson, J., Collins, T., and Bush, H., "A History of Astronaut Construction of Large Space Structures at NASA Langley Research Center," IEEE Aerospace Conference Proceedings, IEEE, New York, 2002, pp. 3569-3587.

[16] Burge, J. H., Sabatke, E., Angel, J. R. P., and Woolf, N. J., "Optical Design of Giant Telescopes for Space," Proceedings of the SPIE, Vol. 4092, Novel Optical Systems Design and Optimization 3, SPIE, Bellingham, WA, 2000, pp. 82-93. doi: $10.1117 / 12.402424$

[17] Jared, R. C., Arthur, A. A., Andreae, S., Biocca, A., Cohen, R. W., Fuertes, J. M., Franck, J., Gabor, G., Llacer, J., Mast, T., Meng, J., Merrick, T., Minor, R., Nelson, J., Orayani, M., Salz, P., Schaefer, B., and Witebsky, C., "The W. M. Keck Telescope Segmented Primary Mirror Active Control System," Proceedings of the SPIE, Vol. 1236, Advanced Technology Optical Telescopes 4, edited by L. D. Barr, SPIE, Bellingham, WA, 1990, pp. 996-1008. doi:10.1117/12.19266

[18] Mast, T., Chanan, G., Nelson, J., Minor, R., and Jared, R., "Edge Sensor Design for the Thirty Meter Telescope," Proceedings of the SPIE, Vol. 6267, Ground-Based and Airborne Telescopes, SPIE, Bellingham, WA, 2006, Paper 62672S. doi: $10.1117 / 12.672028$

[19] Luong, B., Néel, C., Périn, A., and Rozière, D., "Inductive Sensors for Controlling Segmented Mirrors: A Full Industrial and Turnkey Product Solution," SPIE, Vol. 7733, Ground-Based and Airborne Telescopes 3, SPIE, Bellingham, WA, 2010, Paper 77332D. doi: $10.1117 / 12.856406$

[20] Chanan, G., MacMartin, D. G., Nelson, J., and Mast, T., "Control and Alignment of Segmented-Mirror Telescopes: Matrices, Modes, and Error Propagation," Applied Optics, Vol. 43, No. 6, 2004, pp. 1223 1232. doi:10.1364/AO.43.001223

[21] MacMynowski, D. G., "Interaction Matrix Uncertainty in Active (and Adaptive) Optics," Applied Optics, Vol. 48, No. 11, 2009, pp. 2105 2114. doi:10.1364/AO.48.002105

[22] MacMynowski, D. G., "Hierarchic Estimation for Control of Segmented-Mirror Telescopes," Journal of Guidance, Control, and Dynamics, Vol. 28, No. 5, 2005, pp. 1072-1076. doi:10.2514/1.15205

[23] Lessard, L., West, M., MacMynowski, D., and Lall, S., "Warm-Started Wavefront Reconstruction for Adaptive Optics," Journal of the Optical Society of America, A, Vol. 25, No. 5, 2008, pp. 1147-1155. doi:10.1364/JOSAA.25.001147

[24] MacMartin, D. G., "Local, hierarchic, and iterative reconstructors for adaptive optics," Journal of the Optical Society of America, A, Vol. 20, No. 6, 2003, pp. 1084-1093. doi:10.1364/JOSAA.20.001084 